\title{
Instrument-Dependent Factors Affecting the Precision in the Atomic Force Microscopy Stiffness Measurement of Nanoscale Liposomes
}

\author{
Yuki Takechi-Haraya, ${ }^{a}$ Yukihiro Goda,${ }^{b}$ Kenichi Izutsu, ${ }^{a}$ and Kumiko Sakai-Kato*,c \\ ${ }^{a}$ Division of Drugs, National Institute of Health Sciences; 3-25-26 Tonomachi, Kawasaki-ku, Kawasaki 210-9501, \\ Japan: ${ }^{b}$ National Institute of Health Sciences; 3-25-26 Tonomachi, Kawasaki-ku, Kawasaki 210-9501, Japan: and \\ ${ }^{c}$ Kitasato University; 5-9-1 Shirokane, Minato-ku, Tokyo 108-8641, Japan. \\ Received January 22, 2020; accepted February 9, 2020
}

\begin{abstract}
The mechanical strength (stiffness) of liposomes affects their cellular uptake efficiency and drug release in drug delivery processes. We recently developed a tip shape evaluation method for improving the precision of liposome stiffness measurement by quantitative imaging (QI)-mode atomic force microscopy (AFM). The present study applied our method to the widely-used AFM instruments equipped for intermittent contact (IC)-mode force curve measurements, and examined instrument-dependent factors that affect the liposome stiffness measurements. We demonstrated that the evaluation of the tip shape for cantilever selection can be applicable to the IC mode as well as the QI mode. With the cantilever selection, the improved precision of the liposome stiffness was obtained when the stiffness of each liposome was determined from the slope in the force-deformation curve by the IC-mode force curve measurement. Further, the stiffness values were found to be similar to that measured by QI-mode measurements. These results indicate that our developed method can be widely used via IC-mode force curve measurements as well as via QI mode. It was also revealed that spatial drift of the cantilever position was instrument-dependent factors which could affect the precision of liposome stiffness measurements in the case of IC-mode force curve measurement. Therefore, in case of stiffness measurement by IC-mode force curve measurement, it is vital to obtain force-deformation curves immediately after imaging a liposome for the precise stiffness measurement of liposomes. These findings will promote the usage of the AFM stiffness measurement method for the characterization of lipid nanoparticlebased drug delivery systems.
\end{abstract}

Key words atomic force microscopy; liposome stiffness; instrument difference; intermittent contact mode

\section{Introduction}

Based on the concept of drug delivery systems, liposomal drug product development has successfully progressed, as evidenced by the recent small interfering RNA (siRNA)-encapsulating liposomal product Onpattro ${ }^{\circledR}$.) Liposomes, which are a representative drug carrier, are typically prepared using various phospholipids such as distearoylphosphatidylcholine (DSPC) and distearoylphosphatidylglycerol (DSPG), together with cholesterol (Chol). ${ }^{1)}$ Controlling the mechanical strength (stiffness) of liposomes is important because it affects their cellular uptake efficiency and drug release in drug delivery processes. $^{2,3)}$ We have established the use of atomic force microscopy (AFM) to observe the morphology of nanoscale liposomes immobilized on a solid substrate in aqueous medium, and thus to directly measure the stiffness of a liposome. ${ }^{4)}$ In this system, the slope of an AFM force-deformation curve of a liposome corresponds to its stiffness. Our previous study also demonstrated that the efficiency of cellular uptake and penetration into multicellular tumor spheroids of liposomes tends to increase with the liposome stiffness, where the stiffness was determined by AFM. ${ }^{5)}$ Thus, AFM would be a practical method for the design and characterization of liposomal drug products.

However, concern remains regarding the precision of stiffness data obtained by AFM because force-deformation curve measurements vary depending upon the AFM cantilever tip shape. ${ }^{6)}$ In order to apply the AFM method to the development and quality control of liposomal drug products, improvement of the precision of stiffness data is the challenge. To address this issue, via evaluation of cantilever tip shapes using an AFM tip characterizer, we have established a method to improve the precision of AFM stiffness measurements of nanoscale liposomes. Namely, the statistical stiffness value (mean \pm standard deviation (S.D.)) should be calculated from three averaged values obtained by using at least three cantilevers that have a tip aspect ratio greater than 2.5 and a quadratic tip shape function with not more than 10 of root mean square error (RMSE). ${ }^{4)}$ This tip shape evaluation method accompanying the AFM technique (hereafter called TSE/AFM) achieved a large improvement for stiffness precision in terms of relative standard deviation when measuring the stiffness of nanoscale DSPC/DSPG/Chol (53/21/26, mol\% ratio) liposomes. $^{4)}$

To promote the use of AFM method for the development of liposomal drug products, it is crucial to accumulate knowledge about the applicability of our TSE/AFM method to other AFM instruments. Depending upon sample characteristics and preparation conditions, there have usually been used three imaging modes of AFM for soft biological samples, namely contact mode (also called static mode), intermittent contact (IC) mode (also called tapping mode, dynamic mode, or amplitude modulation mode), and jumping mode.,8) The tip shape evaluation and liposome stiffness measurement in our developed TSE/AFM method were established using the quantitative imaging (QI) mode ${ }^{\mathrm{TM}}$ of an AFM, which is a jumping mode available in a JPK microscope. ${ }^{4)}$ The advantage of the 
QI mode is its ability to simultaneously measure a force versus deformation curve at each pixel of each AFM image while imaging the sample surface. ${ }^{9)}$ On the other hand, the imaging mode available on typically-used AFM instruments is the IC mode, which reduces the disruptive influence of lateral forces and induces less deformation on soft samples compared to the contact mode. ${ }^{10)}$ When obtaining liposome stiffness measurements via AFM using IC mode, immediately after imaging the liposomes the cantilever position must be set to the center of a liposome, whereupon the force versus deformation can be obtained by pressing the cantilever onto the surface of the liposome (hereafter referred to as IC-mode force curve measurement). The cantilever motion of this method is different from that in QI mode. ${ }^{9)}$ However, it is unclear whether our developed TSE/AFM method using AFM imaging of a tip characterizer to evaluate cantilever tip shapes used for liposome stiffness measurements can be applicable to the IC mode.

The present study investigated whether our TSE/AFM method can be applicable to widely-used AFM instruments. To this end, the stiffness of DSPC/DSPG/Chol (53/21/26) liposomes was measured via IC-mode force curve measurements. Furthermore, instrument-dependent factors that affect the stiffness of nanoscale liposomes via IC-mode force curve measurement were examined.

\section{Experimental}

Liposome Preparation DSPC/DSPG/Chol (53/21/26, $\mathrm{mol} \%$ ratio) liposomes were prepared as described in our previous study. ${ }^{4)}$ DSPC was purchased from Avanti Polar Lipids (Alabaster, AL, U.S.A.). Chol was purchased from SigmaAldrich (St. Louis, MO, U.S.A.). DSPG was purchased from Cayman Chemical Company (Ann Arbor, MI, U.S.A.). Briefly, the total lipid $(15 \mu \mathrm{mol})$ was dissolved in chloroform-methanol $(2: 1 \mathrm{v} / \mathrm{v})$ in a round-bottomed flask, and the mixture was dried at $60^{\circ} \mathrm{C}$ evaporation to create a thin homogeneous lipid film. The lipid film was further dried overnight by means of vacuum desiccation to remove any residual solvent. The resultant lipid film was hydrated with $5 \%(\mathrm{w} / \mathrm{w})$ glucose aqueous solution ( $\mathrm{pH}$ 5.3), so that the final total lipid concentration was $10 \mathrm{mM}$, under mechanical agitation at $60^{\circ} \mathrm{C}$ for $10 \mathrm{~min}$. The resultant dispersions were freeze-thawed 5 times using a dry ice-methanol slush $\left(-78^{\circ} \mathrm{C}\right)$ and a water bath $\left(60^{\circ} \mathrm{C}\right)$, followed by extrusion 21 times at $60^{\circ} \mathrm{C}$ through a Mini-extruder (Avanti Polar Lipids) equipped with a $100-\mathrm{nm}$ polycarbonate filter. The hydrodynamic diameter and polydispersity of the liposomes were measured at $25^{\circ} \mathrm{C}$ with dynamic light scattering combined with cumulant analysis using a Zetasizer Nano-ZS instrument equipped with Zetasizer Software v.6.01 (Malvern Instruments, Malvern, U.K.). For the measurement, the liposomal suspension was diluted to $0.2 \mathrm{mM}$ with $5 \%(\mathrm{w} / \mathrm{w})$ aqueous glucose solution, and a value of the mean diameter \pm S.D. was obtained from three measurements. The liposome diameter was around $140 \mathrm{~nm}$ and the polydispersity index was $<0.1$, indicating monodispersity.

Tip Shape Evaluation Using AS100P-D Silicon Grating The tip shape evaluation was conducted according to our previous procedure. ${ }^{4)}$ An AS100P-D silicon grating (NTT Advanced Technology, Kanagawa, Japan) with a line and space structure anisotropically etched on a silicon (110) surface was used to characterize the tip shape of a BioLever mini cantilever (BL-AC40TS, Olympus Co., Tokyo, Japan) via $\mathrm{AFM}$ imaging at room temperature $\left(25 \pm 1^{\circ} \mathrm{C}\right)$. The cantilevers had a $0.09 \mathrm{~N} / \mathrm{m}$ nominal spring constant, a nominal tip radius $<15 \mathrm{~nm}$, and a nominal tip angle $<35^{\circ}$. Because of the fabrication process for the AS100P-D grating, the sidewall angles of the line structures were assumed to be exactly $90^{\circ}{ }^{11)}$ The AS100P-D was mounted on a slide glass with double-stick tape and AFM images of the line structures with $52 \pm 2.1 \mathrm{~nm}$ widths and $125 \pm 25 \mathrm{~nm}$ heights (manufacturer specifications) were obtained in air at $25^{\circ} \mathrm{C}$ by means of the QI mode ${ }^{\mathrm{TM}}$ of a JPK Nanowizard Ultra Speed microscope, equipped with the JPK Data Processing Software v.6.0 (JPK Instruments AG, Berlin, Germany). The AFM imaging was conducted at a set point of $0.10 \mathrm{nN}$, a $300 \mathrm{~nm} \mathrm{z}$-length, and an extend/ retract speed of $150 \mu \mathrm{m} / \mathrm{s}$. When using the SHIMADZU SPM-9700 microscope equipped with the SPM software v.4.00 (Shimadzu Corp., Kyoto, Japan), the AS100P-D was mounted on a magnet disk (Shimadzu Corp.) with double-stick tape, and AFM images of the line structures were obtained in air at $25^{\circ} \mathrm{C}$ by means of IC mode with the following scanning parameters: free oscillation amplitude, $1-6 \mathrm{~V}$; operating point, approx. $80 \%$ of oscillation amplitude; drive frequency, $97-131 \mathrm{kHz}$; and scan rate, $1 \mathrm{~Hz}$. For AFM imaging in water, the line sample surface was wetted by applying MilliQ water. The AFM imaging by IC mode was conducted at the following scanning parameters: free oscillation amplitude, $0.1-0.2 \mathrm{~V}$; operating point, approx. $80 \%$ of oscillation amplitude; drive frequency, 22-36 kHz; and scan rate, $1 \mathrm{~Hz}$. All AFM images were obtained at a resolution $<1 \mathrm{~nm} /$ pixel. To correct for sample tilt, the image was flattened via the software and the two-dimensional cross-sectional profile of each line structure was obtained, where the profile was affected by the tip shape. To reconstruct the tip shape, $52 \mathrm{~nm}$ of the line width (the manufacturer's specification) was subtracted from the measured profile, and then the two sections of the profile were aligned. Data at $<10 \%$ of the maximum height of the line structure were excluded because of uncertainty from the bottom of the line structures. The tip shape function (tip length vs. width) was thus derived. In the tip shape function, data at $<2.1 \mathrm{~nm}$ could be obtained; however, these plots had an uncertainty corresponding to an error of $2.1 \mathrm{~nm}$ for the line structures of AS100-PD (the manufacturer's specification). Therefore, we excluded the data at $<2.1 \mathrm{~nm}$. The aspect ratio (tip length/tip width) was determined at the maximum tip width. The average measured value (mean \pm S.D.) was obtained from the analysis of more than three line structures.

Preparation of Liposome Samples for AFM Experiment Liposome samples for AFM measurement were prepared basically according to our previous procedure. ${ }^{4,12)}$ Mica discs (highest quality of Grade V-1, $12 \mathrm{~mm}$ diameter, thickness: $0.15 \mathrm{~mm}$ ) for AFM measurement were purchased from SPI Supplies (West Chester, PA, U.S.A.). A mica disk was glued onto a Shimadzu Petri dish-type aqueous solution cell with silicon adhesive (referred to as "container A"). Immediately after cleavage of the mica, the surface was incubated with $150 \mu \mathrm{L}$ of $0.3 \% \quad(\mathrm{v} / \mathrm{v}) \quad 3$-aminopropyltriethoxysilane (Tokyo Chemical Industry, Tokyo, Japan) aqueous solution for $20 \mathrm{~min}$. 3-Aminopropyltriethoxysilane was purchased from Tokyo Chemical Industry. After the incubation, the surface was rinsed with Milli-Q water and dried in air, resulting in a mica functionalized with 3-aminopropyltriethoxysilane (AP-mica). Then, $150 \mu \mathrm{L}$ of $50 \mu \mathrm{M}$ liposome suspension in $5 \%(w / w)$ glu- 
cose aqueous solution was incubated on the AP-mica substrate for $20 \mathrm{~min}$, and an additional $400 \mu \mathrm{L}$ of $5 \%$ (w/w) glucose aqueous solution was added to the container A, followed by AFM measurement.

AFM Measurement of Liposome Stiffness in Aqueous Medium The stiffness of the liposomes was determined in $5 \%(\mathrm{w} / \mathrm{w})$ aqueous glucose solution $(\mathrm{pH} 5.3)$ at $25 \pm 1^{\circ} \mathrm{C}$ by means of IC-mode force curve measurements via the SHIMADZU microscope, where the method used was basically the same as described in our previous study.,12) Prior to measurements, a tip-evaluated cantilever was calibrated in the air by the thermal noise method using the JPK microscope, ${ }^{13,14)}$ and was immersed in the liquid sample for about $20 \mathrm{~min}$ to obtain thermal equilibrium. The cantilever deflection/photovoltage ratio was calibrated on the AP-mica, and tip cleanliness was checked by monitoring the force curve at the substrate, as previously reported. ${ }^{6}$ The AFM images, with a resolution of less than $8 \mathrm{~nm} /$ pixel, were recorded with the following scanning parameters: free oscillation amplitude, $0.1-1.0 \mathrm{~V}$; operating point, approx. $75-90 \%$ of oscillation amplitude; drive frequency, $22-36 \mathrm{kHz}$; and scan rate, $1 \mathrm{~Hz}$. The force between the tip and the sample was carefully maintained to avoid collapse of the liposome. To correct for sample tilt, the obtained AFM images were subjected to a flattening algorithm by the SHIMADZU software. The liposomes in the AFM images were then extracted using the SHIMADZU Particle Analysis software v.4.00, from which the maximum height of each liposome was determined. ${ }^{15)}$

All force curve measurements were performed by manipulating the cantilever at a velocity of $2 \mu \mathrm{m} / \mathrm{s}$. Immediately after AFM imaging of the liposomes, the cantilever position was set at the center of a liposome, whereupon the force versus deformation curve was obtained by pressing the cantilever to the liposome surface. During this manipulation, each force curve measurement had a dead time of 30-60 s after setting the cantilever position at the liposome center. Stiffness was analyzed by performing a linear fit in the linear region of each curve, where the liposome stiffness corresponds to the value of the slope of the curve. ${ }^{16)}$ For this analysis, the SHIMADZU software was used for the AFM data. Smaller liposomes possess greater stiffness because the membrane has a higher curvature. ${ }^{16)}$ Thus, we confirmed that comparison groups analyzed by AFM exhibited no significant differences in height distribution, as previously described. ${ }^{4)}$ For each sample, the averaged stiffness data were obtained with at least 9 liposomes for every cantilever.

Statistical Analysis Data were expressed as the mean \pm S.D. Results were analyzed by the Student's $t$-test. Differences were considered statistically significant at $p<0.05$.

\section{Results and Discussion}

Cantilever tip shape evaluation is an essential part of our TSE/AFM method, and the cantilever selection for liposome stiffness measurement can be done according to the criteria (i.e., a tip aspect ratio greater than 2.5 and a quadratic tip shape function with not more than 10 of root-mean-square error (RMSE)) by analyzing the tip shape function derived from AFM image analysis of line structures, as previously described. ${ }^{4)}$ This TSE/AFM method is useful because commercial line samples of tip characterizers can be used, the measurement can be done with a single scan, and complex algorithms or software are not needed. ${ }^{4,11)}$ Our previous study has shown the importance of the TSE/AFM method when using QI mode, but widely-used AFM instruments in biomedical applications are equipped for IC mode., ${ }^{47}$ ) To investigate the applicability of the TSE/AFM method, the line sample was scanned via IC mode of a SHIMADZU microscope (Fig. 1). Stable scanning was impossible using a BioLever Mini cantilever, which possesses a low nominal spring constant of

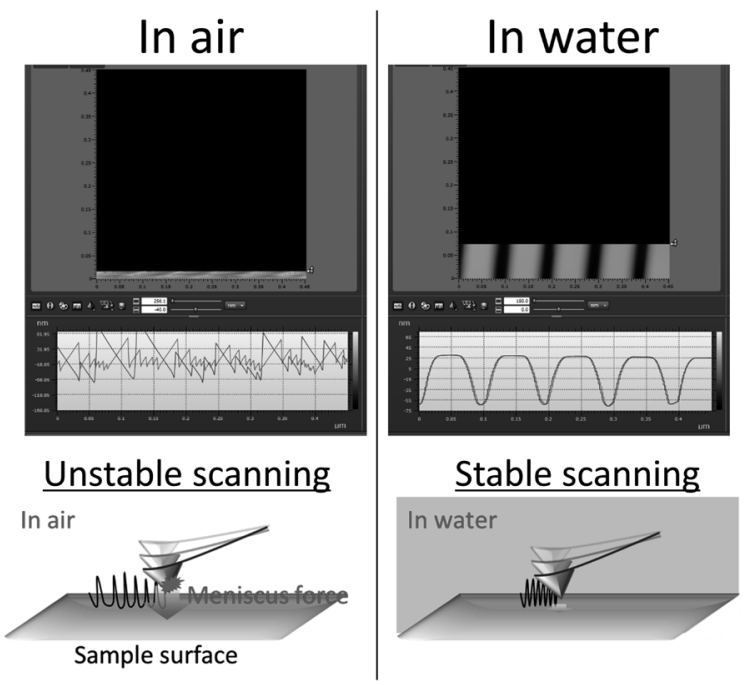

Fig. 1. Schematic of AFM Scanning of AS100P-D Silicon Grating Line Structures in Air (Left Panel) and in Water (Right Panel) via IC Mode

In air, the meniscus force between the cantilever tip and sample surface was significant, resulting in unstable scanning (left panel). In water, the scanning was stable because of the decreased meniscus force (right panel).

(A)

\section{Cantilever \#1}
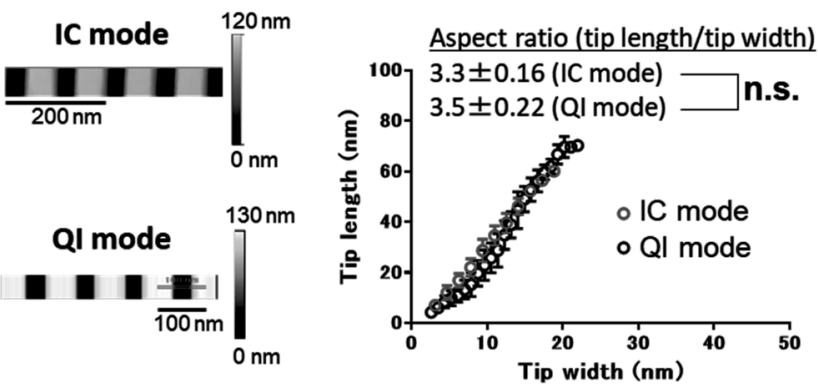

(B)

\section{Cantilever\#2}
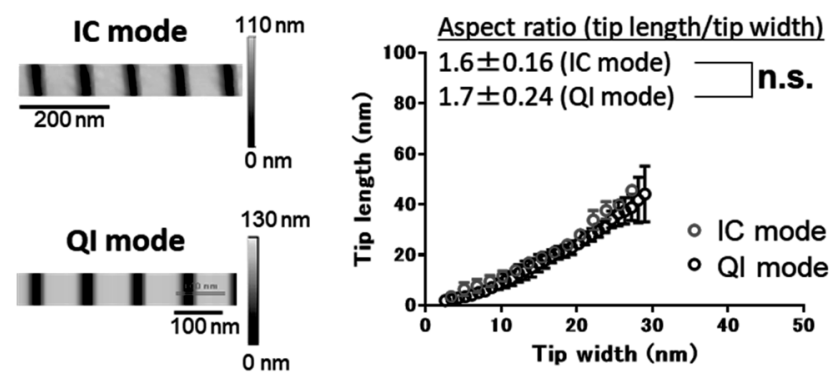

Fig. 2. Differences in Scanning Mode for IC Mode (SHIIMADZU) and QI Mode (JPK) during Evaluation of Cantilever Tip Shapes for (A) Sharp Cantilever \#1 and (B) Blunt Cantilever \#2

Tip shape analysis included imaging the AS100P-D silicon grating line structures and analyzing the tip shape functions. 
(A)

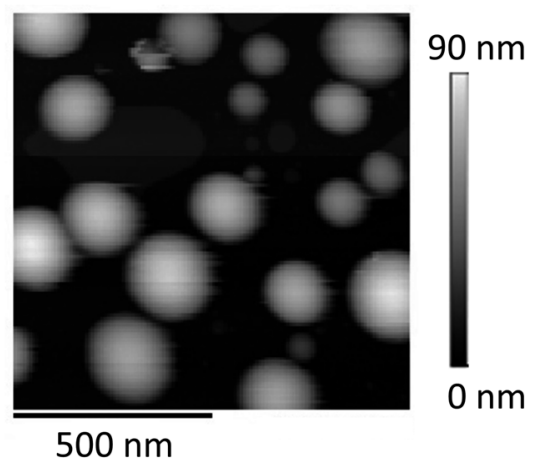

(B)

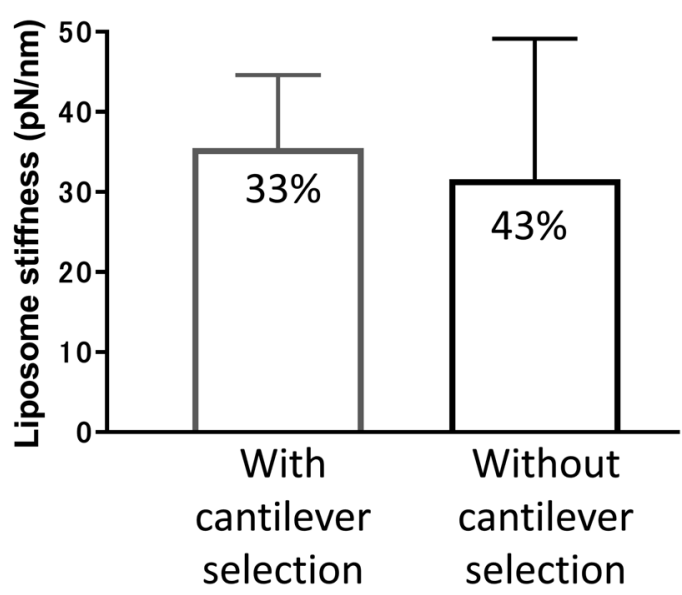

Fig. 3. Stiffness Measurements of DSPC/DSPG/Chol (53/21/26) Liposomes on AP-Mica in $5 \%(\mathrm{w} / \mathrm{w})$ Aqueous Glucose Solution at $25^{\circ} \mathrm{C}$ by Means of IC-Mode Force Curve Measurement

(A) AFM images of liposomes. (B) The statistical liposome stiffness (means \pm standard deviations (S.D.s) with and without cantilever selection. The percentage shown in each column shows the relative standard deviation. Heights (means \pm S.D.s) of liposomes for the data with and without cantilever selection were $77 \pm 10$ and $79 \pm 10 \mathrm{~nm}$, respectively.
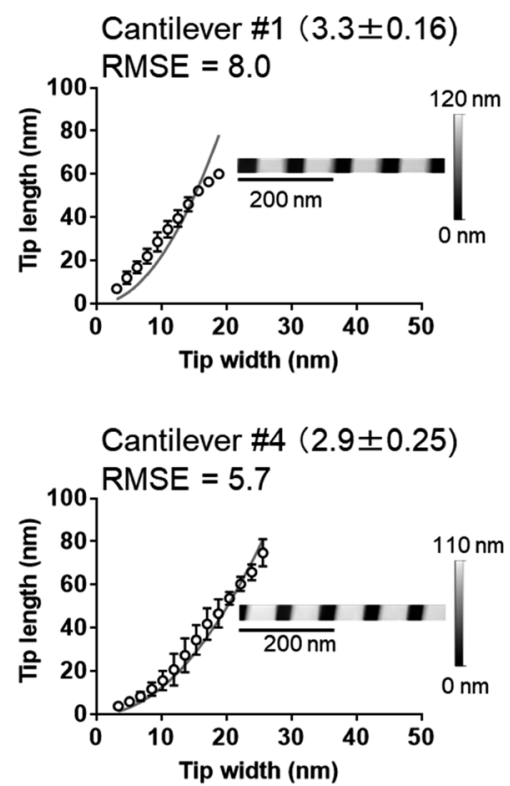

$0.09 \mathrm{~N} / \mathrm{m}$, and no clear AFM images were obtained (Fig. 1, left panel). It was considered that IC-mode scanning with a soft cantilever is disturbed by the large meniscus force produced by the absorbed water nano-meniscus between the cantilever tip and the substrate in air. ${ }^{18)}$ To solve this problem, we conducted AFM imaging of the line samples in water, thereby mitigating the meniscus force and establishing stable scanning of the line structures by IC mode (Fig. 1, right panel).

Thus, the TSE/AFM method via IC mode of the SHIMADZU microscope was compared to that via QI mode of a JPK microscope (Fig. 2). The two AFM modes produced similar AFM images and tip shape functions, resulting in no significant differences of tip aspect ratios either when using a cantilever with a sharp tip (Cantilever \#1, Fig. 2A) or with a blunt tip (Cantilever \#2, Fig. 2B). This result demonstrates that the TSE/AFM method using the QI mode for evaluating the tip shape of cantilevers can be applicable even with the widely-used IC mode.

Furthermore, with cantilever selection, the spherical structure of DSPC/DSPG/Chol (53/21/26) liposomes were easily observed by IC mode (Fig. 3A), and improved precision of the liposomes stiffness was obtained when the stiffness of each liposomes was determined from the slope in the forcedeformation curve obtained by the IC-mode force curve measurement (Fig. 3B). In this case, three cantilevers satisfying the cantilever screening criteria were used (Fig. 4). Also, the obtained stiffness value in Fig. 3B exhibited no significant difference compared to that for the same liposomes obtained by TSE/AFM in QI mode in our previous study (32 pN/nm), in which measurement the method validity was confirmed. ${ }^{4}$ These results clarify that the TSE/AFM method can be widely used via the IC-mode force curve measurement as well as via the QI mode.

Although similar liposome stiffness values were obtained by the two different measurement mode, there still exists some variations in the stiffness value obtained by the IC-

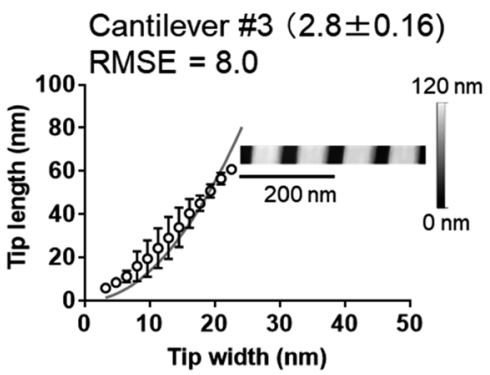

Fig. 4. Evaluation of AFM Cantilever Tip Shapes by Using Line Structures of the AS100P-D Silicon Grating

Each panel shows the tip shape function and corresponding AFM image. The data for Cantilever \#1 was from Fig. 2A. The numerical values (mean \pm S.D.) represent the tip aspect ratios at maximum tip width. The lines are the best fit curves to the experimental data using a quadratic function $\left(\right.$ Tip length $=$ constant $\times$ Tip width ${ }^{2}$. Root mean squared errors (RMSE) with regard to the fitting were also shown. 
(A)

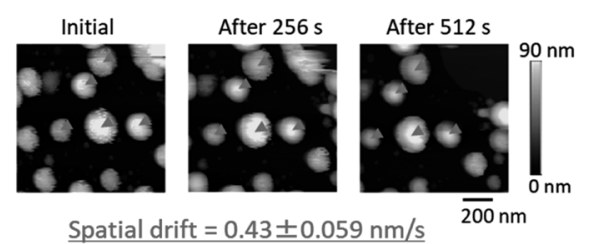

(B)

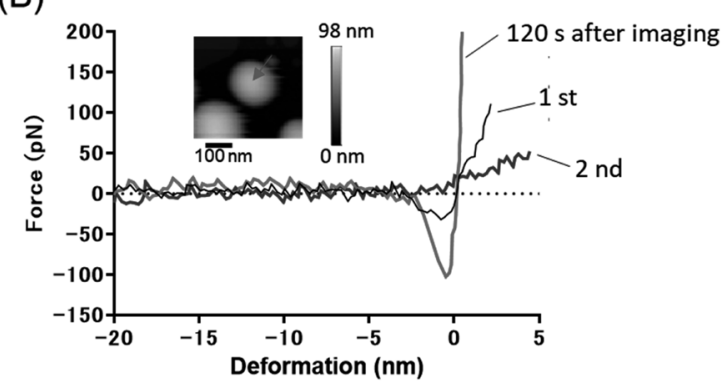

(C)

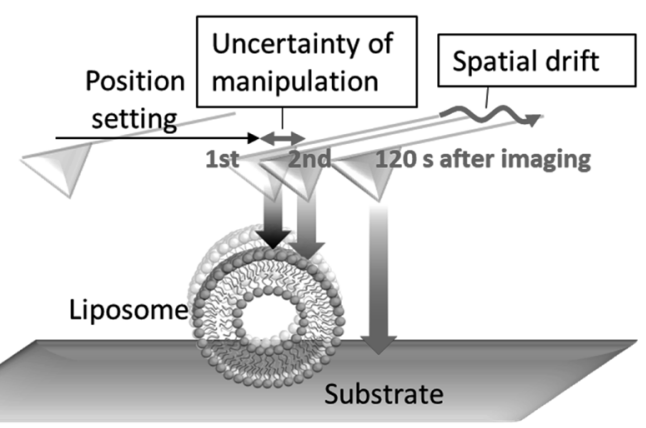

Fig. 5. Spatial Drift and Cantilever Manipulation Uncertainty in the $x-y$ Direction, and the Effect of These on the AFM Stiffness Measurements for DSPC/DSPG/Chol (53/21/26) Liposomes on AP-Mica in $5 \%$ (w/w) Aqueous Glucose Solution at $25^{\circ} \mathrm{C}$

(A) AFM images obtained by IC mode at the same $x-y$ piezoelectric actuator setting at three different times. The spatial drift was calculated by tracing the $x-y$ coordinates of the liposome centers indicated by arrows. (B) Location precision of the force-deformation curve measurement of a liposome via IC-mode force curve measurement. The liposome indicated by an arrow in the AFM image (inset) was analyzed twice, where each curve (1st and 2 nd) was obtained immediately after imaging the liposome and centering the cantilever. To determine the effect of spatial drift, a force curve was also obtained $120 \mathrm{~s}$ after the second imaging and centering the cantilever. (C) Schematic representation of the mechanism governing variation of the force-deformation curves.

mode force curve measurement compared to the QI-mode force curve measurement. ${ }^{4)}$ It should be noted that the stiffness measurement by IC-mode force curve measurement was obtained as follows (see also Experimental): immediately after the AFM imaging of liposomes, the cantilever position, which is controlled by a piezoelectric actuator, was set at the center of a liposome, whereupon the force-deformation curve was obtained by pressing the cantilever to the liposome surface. During this manipulation, each force-deformation curve measurement had a dead time of $30-60 \mathrm{~s}$ after setting the cantilever position at the liposome center via the piezoelectric actuator. Thus, the spatial drift of the cantilever position via a piezoelectric actuator would cause a displacement in the $x-y$ direction from the center position, affecting the precision of the liposome stiffness measurement.

Hence, we investigated the $x-y$ spatial drift by tracing the $x-y$ position of each liposome center as a function of time after setting the piezoelectric actuator at a fixed $x-y$ position (Fig. 5A). As a result, the spatial drift value was found to be
$0.43 \mathrm{~nm} / \mathrm{s}$. Indeed, when measuring a force-deformation curve $120 \mathrm{~s}$ after setting the cantilever position at the center of a liposome, the curve reflected not the liposome but the substrate (Figs. 5B, C). Moreover, the force-deformation curve measurements were independently repeated twice on a single liposome while targeting the center of that liposome (Fig. 5B, 1st and 2 nd curves). It was found that the repeated measurement gave a variation in the force-deformation curve. A previouslyreported theoretical and experimental study demonstrated that the stiffness of a liposome decreases from the center toward the edge of the liposome owing to the lack of support by the liposome surface. ${ }^{6)}$ Thus, we can observe that, although the first force-deformation curve was obtained at the center of a liposome, the second one was actually obtained near the liposome edge despite setting the cantilever position at the liposome center (Fig. 5C). This result indicates that, during the stiffness measurement, there possibly exists an uncertainty of the cantilever (piezoelectric actuator) manipulation. Taken together, in case of stiffness measurement by IC-mode force curve measurement, it is vital to obtain force-deformation curves immediately after imaging a liposome for the precise stiffness measurement of liposomes.

\section{Conclusion}

We showed that the previously-developed TSE/AFM method is applicable to the widely-used IC-mode force curve measurement. There was no significant difference between the averaged liposome stiffness values obtained by IC-mode force curve measurement and QI-mode. Furthermore, the TSE/AFM method was valid in the widely-used IC-mode force curve measurements and could improve the precision of the obtained values. It was also found that it is vital to obtain force-deformation curves immediately after imaging a liposome for the precise stiffness measurement of liposomes. Because of the increasing impact of nanoparticle stiffness upon nanoparticlebased drug delivery systems, ${ }^{19)}$ these findings will promote the usage of the AFM stiffness measurement method for the development and quality control of lipid nanoparticle-based drug delivery systems.

Acknowledgments This work was supported in part by the Research on Regulatory Harmonization and Evaluation of Pharmaceuticals, Medical Devices, Regenerative and Cellular Therapy Products, Gene Therapy Products, and Cosmetics from the Japan Agency for Medical Research and Development, AMED (No. 19mk0101082j0103).

Conflict of Interest The authors declare no conflict of interest.

\section{References}

1) Leung A. W. Y., Amador C., Wang L. C., Mody U. V., Bally M. B., Pharmaceutics, 11, 1-26 (2019).

2) Zhang S., Gao H., Bao G., ACS Nano, 9, 8655-8671 (2015).

3) Lee J., Kim J., Jeong M., Lee H., Goh U., Kim H., Kim B., Park J. H., Nano Lett., 15, 2938-2944 (2015).

4) Takechi-Haraya Y., Goda Y., Izutsu K., Sakai-Kato K., Anal. Chem. 91, 10432-10440 (2019).

5) Takechi-Haraya Y., Goda Y., Sakai-Kato K., Mol. Pharm., 14, 2158-2165 (2017)

6) Vorselen D., Mackintosh F. C., Roos W. H., Wuite G. J. L., ACS 
Nano, 11, 2628-2636 (2017).

7) Moreno-Herrero F., Colchero J., Gómez-Herrero J., Baró A. M., Phys. Rev. E Stat. Nonlin. Soft Matter Phys., 69, 031915 (2004).

8) Gan Y., Surf. Sci. Rep., 64, 99-121 (2009).

9) Smolyakov G., Formosa-Dague C., Severac C., Duval R. E., Dague E., Micron, 85, 8-14 (2016).

10) Putman C. A. J., Van Der Werf K. O., De Grooth B. G., Van Hulst N. F., Greve J., Appl. Phys. Lett., 64, 2454-2456 (1994).

11) Takenaka H., Hatayama M., Ito H., Ohchi T., Takano A., Kurosawa S., Itoh H., Ichimura S., J. Surf. Anal., 17, 264-268 (2011).

12) Takechi-Haraya Y., Sakai-Kato K., Abe Y., Kawanishi T., Okuda H., Goda Y., Langmuir, 32, 6074-6082 (2016).
13) Hutter J. L., Bechhoefer J., Rev. Sci. Instrum., 64, 1868-1873 (1993).

14) Sader J. E., Chon J. W. M., Mulvaney P., Rev. Sci. Instrum., 70, 3967-3969 (1999).

15) Nečas D., Klapetek P., Cent. Eur. J. Phys., 10, 181-188 (2012).

16) Delorme N., Fery A., Phys. Rev. E Stat. Nonlin. Soft Matter Phys., 74, 030901 (2006).

17) Maver U., Velnar T., Gaberšček M., Planinšek O., Finšgar M., Trends Analyt. Chem., 80, 96-111 (2016).

18) Butt H. J., Cappella B., Kappl M., Surf. Sci. Rep., 59, 1-152 (2005). 19) Anselmo A. C., Mitragotri S., Adv. Drug Deliv. Rev., 108, 51-67 (2017). 